\title{
Multi-utility representations of incomplete preferences induced by set-valued risk measures
}

\author{
Cosimo Munari ${ }^{1}$
}

Received: 31 October 2019 / Accepted: 3 August 2020

(C) The Author(s) 2020

\begin{abstract}
We establish a variety of numerical representations of preference relations induced by set-valued risk measures. Because of the general incompleteness of such preferences, we have to deal with multi-utility representations. We look for representations that are both parsimonious (the family of representing functionals is indexed by a tractable set of parameters) and well behaved (the representing functionals satisfy nice regularity properties with respect to the structure of the underlying space of alternatives). The key to our results is a general dual representation of set-valued risk measures that unifies the existing dual representations in the literature and highlights their link with duality results for scalar risk measures.
\end{abstract}

Keywords Risk measures · Dual representations · Incomplete preferences · Multi-utility representations

Mathematics Subject Classification (2010) 91B06 · 91G80

JEL Classification C60 - G11

\section{Introduction}

This note is concerned with the numerical representation of preference relations induced by a special class of set-valued maps. Recall that a preference (relation) over the elements of a set $L$ is a reflexive and transitive binary relation on $L$. A preference is said to be complete if any two elements $x, y \in L$ are comparable in the sense that

C. Munari

cosimo.munari@bf.uzh.ch

1 Center for Finance and Insurance and Swiss Finance Institute, Department of Banking and Finance, University of Zurich, Plattenstrasse 14, 8032 Zurich, Switzerland 
it is always possible to determine whether $x$ is preferred to $y$ or vice versa. Following the terminology of Dubra et al. [14], a family $\mathcal{U}$ of maps $u: L \rightarrow[-\infty, \infty]$ is a multi-utility representation of a preference $\succeq$ if for all $x, y \in L$, we have

$$
x \succeq y \quad \Longleftrightarrow \quad u(x) \geq u(y) \text { for every } u \in \mathcal{U} .
$$

In words, a multi-utility representation provides a numerical representation for the given preference relation via a family of "utility functionals". In view of their greater tractability, multi-utility representations play a fundamental role in applications. A standard problem in this context is to find representations that are at the same time parsimonious (the family of representing functionals is indexed by a small set of parameters) and well behaved (the representing functionals satisfy nice regularity properties with respect to the structure of the underlying set). This is especially important for incomplete preferences, which cannot be represented by a unique functional.

In the broad field of economics and finance, incomplete preferences arise naturally in the presence of multi-criteria decision making where agents are often unable to prioritise the different criteria, thereby failing to arrive at a definitive preference comparison between all possible alternatives. Classical works investigate multi-utility representations of preferences over sets of consumption bundles or lotteries, but there exists a considerable literature focusing on preferences defined on abstract sets by means of suitable axioms. We refer to Aumann [5] and Bewley [8] for two classical references and to Ok [39], Dubra et al. [14], Mandler [36], Eliaz and Ok [15], Kaminski [34], Evren [16, 17], Evren and Ok [18], Bosi and Herden [10], Galaabaatar and Karni [26], Nishimura and Ok [38], Bosi et al. [9] and Bevilacqua et al. [7] for an overview of contributions to the theory of incomplete preferences and their multiutility representations in the last 20 years.

The goal of this note is to establish numerical representations of preference relations induced by a special class of set-valued maps that have been the subject of intense research in the recent mathematical finance literature. To introduce the underlying economic problem, consider an economic agent who is confronted with the problem of ranking a number of different alternatives represented by the elements of a set $L$. The agent has specified a target set of acceptable or attractive alternatives $A \subseteq L$. We assume that if an alternative is not acceptable, it can be made acceptable upon implementation of a suitable admissible action. We represent the results of admissible actions by the elements of a set $M \subseteq L$ and assume that a given alternative $x \in L$ can be transformed through a given $m \in M$ into the new alternative $x+m$. The objective of the agent is then to identify, for each alternative, all the admissible actions that can be implemented to move said alternative inside the target set by way of translations. This naturally leads to the set-valued map $R: L \rightrightarrows M$ defined by

$$
R(x):=\{m \in M: x+m \in A\}=M \cap(A-x) .
$$

Here, we used the standard double arrow notation $\rightrightarrows$ to denote a map taking values in the power set of $M$. The map $R$ can be seen as a generalisation of the set-valued risk measures studied by Jouini et al. [32], Kulikov [35], Hamel and Heyde [28], Hamel et al. [30] and Molchanov and Cascos [37] in the context of markets with transaction costs; by Haier et al. [27] in the context of intragroup transfers; by Feinstein 
et al. [22], Armenti et al. [3] and Ararat and Rudloff [1] in the context of systemic risk. We refer to these contributions for a discussion about the financial interpretation of set-valued risk measures in the respective fields of application and to Sect. 5 for some concrete examples in the context of multi-currency markets with transaction costs and systemic risk.

The set-valued map $R$ defined above induces a preference relation on $L$ by setting

$$
x \succeq_{R} y \quad: \Longleftrightarrow \quad R(x) \supseteq R(y) .
$$

According to this preference, the agent prefers $x$ to $y$ if every admissible action through which we can move $y$ into the target set will also allow us to transport $x$ there. In other terms, $x$ is preferred to $y$ if it is easier to make $x$ acceptable compared to $y$. The goal of this note is to establish numerical representations of the preference $\succeq_{R}$. Since this preference, as shown below, is not complete in general, we have to deal with multi-utility representations. In particular, we look for representations consisting of (semi)continuous utility functionals. We achieve this by establishing suitable (dual) representations of the set-valued map $R$.

Our results provide a unifying perspective on the existing dual representations of set-valued risk measures and on the corresponding multi-utility representations, which to be best of our knowledge have never been explicitly investigated in the literature. We illustrate the advantages of such a unifying approach by discussing applications to multi-currency markets with transaction costs and systemic risk. In addition, we highlight where our strategy to establishing dual representations differs from the standard arguments used in the literature.

The rest of the note is structured as follows. The necessary mathematical background is collected in Sect. 2. The standing assumptions on the space of alternatives and the main properties of the set-valued map under investigation are presented in Sect. 3. The main results on dual and multi-utility representations are established in Sect. 4 and are applied to a number of concrete situations in Sect. 5.

\section{Mathematical background}

In this section, we collect the necessary mathematical background and fix the notation and terminology used throughout the paper. We refer to Rockafellar [40, Sects. 1 and 3] and Zălinescu [43, Chap. 2] for a thorough presentation of duality for topological vector spaces. Moreover, we refer to Aubin and Ekeland [4, Chap. 1] for a variety of results on support functions and barrier cones.

Let $L$ be a real locally convex Hausdorff topological vector space. The topological dual of $L$ is denoted by $L^{\prime}$. Any linear subspace $M \subseteq L$ is canonically equipped with the relative topology inherited from $L$. The corresponding dual space is denoted by $M^{\prime}$. For every set $A \subseteq L$, we denote by $\operatorname{int}(A)$ and $\operatorname{cl}(A)$ the interior and the closure of $A$, respectively. We say that $A$ is convex if $\lambda A+(1-\lambda) A \subseteq A$ for every $\lambda \in[0,1]$, and that $A$ is a cone if $\lambda A \subseteq A$ for every $\lambda \in[0, \infty)$. The (lower) support function of $A$ is the map $\sigma_{A}: L^{\prime} \rightarrow[-\infty, \infty]$ defined by

$$
\sigma_{A}(\psi):=\inf _{x \in A} \psi(x) .
$$


The effective domain of $\sigma_{A}$ is called the barrier cone of $A$ and is denoted by

$$
\operatorname{bar}(A):=\left\{\psi \in L^{\prime}: \sigma_{A}(\psi)>-\infty\right\} .
$$

It follows from the Hahn-Banach theorem that if $A$ is closed and convex, then it can be represented as the intersection of all the halfspaces containing it, or equivalently

$$
A=\bigcap_{\psi \in L^{\prime}}\left\{x \in L: \psi(x) \geq \sigma_{A}(\psi)\right\}=\bigcap_{\psi \in \operatorname{bar}(A)}\left\{x \in L: \psi(x) \geq \sigma_{A}(\psi)\right\} .
$$

If $A$ is a cone, then $\operatorname{bar}(A)$ coincides with the polar or dual cone of $A$, i.e.,

$$
\operatorname{bar}(A)=A^{+}:=\left\{\psi \in L^{\prime}: \psi(x) \geq 0, \forall x \in A\right\} .
$$

If $A$ is a vector space, then $\operatorname{bar}(A)$ coincides with the annihilator of $A$, i.e.,

$$
\operatorname{bar}(A)=A^{\perp}:=\left\{\psi \in L^{\prime}: \psi(x)=0, \forall x \in A\right\} .
$$

Finally, if $A+K \subseteq A$ for some cone $K \subseteq L$, then $\operatorname{bar}(A) \subseteq K^{+}$.

\section{The setting}

Throughout the remainder of the paper, we assume that $L$ is a real locally convex Hausdorff topological vector space. We also fix a closed convex cone $K \subseteq L$ satisfying $K-K=L$ and consider the induced partial order defined by

$$
x \succeq_{K} y \quad: \Longleftrightarrow \quad x-y \in K .
$$

The above partial order is meant to capture an "objective" preference relation shared by all agents. This is akin to the "better for sure" preference in Drapeau and Kupper [13].

Assumption 3.1 Recall (1.1). We stipulate the following assumptions on $A$ and $M$ :

(A1) $A$ is closed, convex and satisfies $A+K \subseteq A$.

(A2) $M$ is a closed linear subspace of $L$ such that $M \cap K \neq\{0\}$.

(A3) $R(x) \notin\{\emptyset, M\}$ for some $x \in L$.

The next proposition collects a number of basic properties of the set-valued map $R$ and its associated preference $\succeq_{R}$. The properties of $R$ are aligned with those discussed in Hamel and Heyde [28] and Hamel et al. [30].

Proposition 3.2 (i) $\succeq_{R}$ is monotone with respect to $K$, i.e., for all $x, y \in L$,

$$
x \succeq_{K} y \quad \Longrightarrow \quad x \succeq_{R} y \text {. }
$$

(ii) $\succeq_{R}$ is convex, i.e., for all $x, y \in L$ and $\lambda \in[0,1]$,

$$
x \succeq_{R} y \quad \Longrightarrow \quad \lambda x+(1-\lambda) y \succeq_{R} y .
$$


(iii) $R(x)+K \cap M \subseteq R(x)$ for every $x \in L$.

(iv) $R(x+m)=R(x)-m$ for all $x \in L$ and $m \in M$.

(v) $R(\lambda x+(1-\lambda) y) \supseteq \lambda R(x)+(1-\lambda) R(y)$ for all $x, y \in L$ and $\lambda \in[0,1]$.

(vi) $R(x)$ is convex and closed for every $x \in L$.

(vii) $R(x) \neq M$ for every $x \in L$.

Proof To establish (i), assume that $x \succeq_{K} y$ for $x, y \in L$. For every $m \in R(y)$,

$$
x+m=y+m+x-y \in A+K \subseteq A .
$$

This shows that $m \in R(x)$ as well, so that $x \succeq_{R} y$. To establish (ii), take $\lambda \in[0,1]$ and assume that $x \succeq_{R} y$. For every $m \in R(y)$, we have $y+m \in A$ and hence $x+m \in A$. This yields

$$
\lambda x+(1-\lambda) y+m=\lambda(x+m)+(1-\lambda)(y+m) \in \lambda A+(1-\lambda) A \subseteq A,
$$

showing that $m \in R(\lambda x+(1-\lambda) y)$. In sum, $\lambda x+(1-\lambda) y \succeq_{R} y$. To see that properties (iii) to (vi) hold, it suffices to recall that $R(x)=M \cap(A-x)$ for every $x \in L$. Finally, to establish (vii), assume that $R(x)=M$ for some $x \in L$. Take any $y \in L$ and assume that $R(y)$ is nonempty so that $y+m \in A$ for some $m \in M$. For all $n \in M$ and $\lambda \in(0,1]$, we have

$$
\lambda\left(x+\frac{1}{\lambda}(n-m)\right)+(1-\lambda)(y+m) \in \lambda A+(1-\lambda) A \subseteq A
$$

by convexity. Hence, letting $\lambda \rightarrow 0$, we obtain $y+n \in A$ by closedness. Since $n$ was arbitrary, we infer that $R(y)=M$. This contradicts Assumption 3.1, (A3), showing that $R(x) \neq M$ must hold for every $x \in L$.

Remark 3.3 (i) If $M$ is spanned by a single element, then $\succeq_{R}$ is complete. Indeed, in this case, we can always assume that $M$ is spanned by a nonzero element $m \in M \cap K$ by our standing assumption. Then, for every $x \in L$ such that $R(x) \neq \emptyset$, we see that

$$
R(x)=\left\{\lambda m: \lambda \in\left[\lambda_{x}, \infty\right)\right\}
$$

for a suitable $\lambda_{x} \in \mathbb{R}$. This shows that $\succeq_{R}$ is complete.

(ii) In general, the preference $\succeq_{R}$ is not complete when $M$ is spanned by more than one element. For instance, let $L=\mathbb{R}^{3}$ and assume that $K=A=\mathbb{R}_{+}^{3}$ and $M=\mathbb{R}^{2} \times\{0\}$. For $x=0$ and $y=(1,-1,0)$, we respectively have

$$
R(x)=\left\{m \in M: m_{1} \geq 0, m_{2} \geq 0\right\} \quad \text { and } \quad R(y)=\left\{m \in M: m_{1} \geq-1, m_{2} \geq 1\right\} .
$$

Clearly, neither $x \succeq_{R} y$ nor $y \succeq_{R} x$ holds, showing that $\succeq_{R}$ is not complete.

(iii) Sometimes the preference $\succeq_{R}$ is complete even if $M$ is spanned by more than one element. For instance, let $L=\mathbb{R}^{3}$ and assume that $K=A=\mathbb{R}_{+}^{2} \times \mathbb{R}$ and $M=\{0\} \times \mathbb{R}^{2}$. For every $x \in L$ such that $R(x) \neq \emptyset$, we have

$$
R(x)=\left\{m \in M: m_{2} \geq-x_{2}\right\} .
$$

This shows that $\succeq_{R}$ is complete. 


\section{Multi-utility representations}

In this section, we establish a variety of multi-utility representations of the preference induced by $R$, which are derived from suitable representations of the sets $R(x)$. As highlighted below, both representations have a strong link with (scalar) risk measures and their dual representations.

The first multi-utility representation is based on the following scalarisations of $R$. Here, we set

$$
K_{M}^{+}:=\left\{\pi \in M^{\prime}: \pi(m) \geq 0, \forall m \in K \cap M\right\} .
$$

Definition 4.1 For every $\pi \in K_{M}^{+}$, we define a map $\rho_{\pi}: L \rightarrow[-\infty, \infty]$ by setting

$$
\rho_{\pi}(x):=\inf \{\pi(m): m \in M, x+m \in A\}=\inf \{\pi(m): m \in R(x)\} .
$$

Moreover, we define a map $u_{\pi}: L \rightarrow[-\infty, \infty]$ by setting

$$
u_{\pi}(x):=-\rho_{\pi}(x) .
$$

The functionals $\rho_{\pi}$ are examples of the risk measures introduced in Föllmer and Schied [23] and generalised in Frittelli and Scandolo [25]. We refer to Farkas et al. $[19,20]$ for a thorough investigation of such functionals at our level of generality. The next proposition features some of their standard properties which follow immediately from Proposition 3.2. Since the announced multi-utility representation will be expressed in terms of the negatives of the functionals $\rho_{\pi}$, the proposition is stated in terms of the utility functionals $u_{\pi}$.

Proposition 4.2 For every $\pi \in K_{M}^{+}$, the functional $u_{\pi}$ satisfies the following properties:

(i) $u_{\pi}$ is translative along $M$, i.e., for all $x \in L$ and $m \in M$,

$$
u_{\pi}(x+m)=u_{\pi}(x)+\pi(m) .
$$

(ii) $u_{\pi}$ is nondecreasing with respect to $\succeq_{K}$, i.e., for all $x, y \in L$,

$$
x \succeq_{K} y \quad \Longrightarrow \quad u_{\pi}(x) \geq u_{\pi}(y) .
$$

(iii) $u_{\pi}$ is concave, i.e., for all $x, y \in L$ and $\lambda \in[0,1]$,

$$
u_{\pi}(\lambda x+(1-\lambda) y) \geq \lambda u_{\pi}(x)+(1-\lambda) u_{\pi}(y) .
$$

Remark 4.3 Note that unless $M$ is spanned by one element, the closedness of the set $A$ is not sufficient to ensure that the functionals $\rho_{\pi}$ are lower semicontinuous; see Farkas et al. [20, Example 1]. We refer to Hamel et al. [29] for a discussion on general sufficient conditions ensuring the lower semicontinuity of scalarisations of set-valued maps and to Farkas et al. [20] and Baes et al. [6] for a variety of sufficient conditions in a risk measure setting. 
The first multi-utility representation of the preference induced by $R$ rests on the intimate link between the risk measures $\rho_{\pi}$ and the support functions corresponding to $R$.

Lemma 4.4 For every $x \in L$, the set $R(x)$ can be represented as

$$
R(x)=\bigcap_{\pi \in K_{M}^{+} \backslash\{0\}}\left\{m \in M: \pi(m) \geq \rho_{\pi}(x)\right\} .
$$

Proof The result is clear if $R(x)=\emptyset$. Otherwise, recall that $R(x)$ is closed and convex by Proposition 3.2 and observe that $\rho_{\pi}(x)=\sigma_{R(x)}(\pi)$ for every $\pi \in M^{\prime}$. We can apply the dual representation (2.1) in the context of the space $M$ to obtain

$$
R(x)=\bigcap_{\pi \in \operatorname{bar}(R(x)) \backslash\{0\}}\left\{m \in M: \pi(m) \geq \rho_{\pi}(x)\right\} .
$$

As $R(x)+K \cap M \subseteq R(x)$ again by Proposition 3.2, we infer that the barrier cone of $R(x)$ must be contained in $K_{M}^{+}$. We can therefore replace $\operatorname{bar}(R(x))$ with $K_{M}^{+}$in the above intersection because $\rho_{\pi}(x)=\sigma_{R(x)}(\pi)=-\infty$ for every $\pi \in M^{\prime} \backslash \operatorname{bar}(R(x))$.

Theorem 4.5 The preference $\succeq_{R}$ can be represented by the multi-utility family

$$
\mathcal{U}=\left\{u_{\pi}: \pi \in K_{M}^{+} \backslash\{0\}\right\} .
$$

Proof We rely on Lemma 4.4. Take any $x, y \in L$. If $x \succeq_{R} y$, then $R(x) \supseteq R(y)$ and

$$
\rho_{\pi}(x)=\sigma_{R(x)}(\pi) \leq \sigma_{R(y)}(\pi)=\rho_{\pi}(y)
$$

for every $\pi \in K_{M}^{+} \backslash\{0\}$. Conversely, if $\rho_{\pi}(x) \leq \rho_{\pi}(y)$ for every $\pi \in K_{M}^{+} \backslash\{0\}$, then for each $m \in R(y)$, we have $\pi(m) \geq \rho_{\pi}(y) \geq \rho_{\pi}(x)$ for every $\pi \in K_{M}^{+} \backslash\{0\}$, so that $m \in R(x)$. This yields $x \succeq_{R} y$ and concludes the proof.

Remark 4.6 The simple representation in Lemma 4.4 shows that the set-valued map $R$ is completely characterised by the family of functionals $\rho_{\pi}$. In the context of risk measures, one could say that a set-valued risk measure is completely characterised by the corresponding family of scalar risk measures; see Definition 4.1. This corresponds to the "setification" formula in Hamel et al. [29, Sect. 4.2].

We aim to improve the above representation in two ways. First, we want to find a multi-utility representation consisting of a smaller number of representing functionals. This is important to ensure a more parsimonious, hence tractable, representation. Second, we want to establish a multi-utility representation consisting of (semi)continuous representing functionals. This is important in applications, e.g. in optimisation problems where the preference appears in the optimisation domain. 
The second multi-utility representation will be expressed in terms of the following utility functionals. Here, for any functional $\pi \in M^{\prime}$, we denote by $\operatorname{ext}(\pi)$ the set of all linear continuous extensions of $\pi$ to the whole space $L$, i.e.,

$$
\operatorname{ext}(\pi):=\left\{\psi \in L^{\prime}: \psi(m)=\pi(m), \forall m \in M\right\}
$$

Definition 4.7 For every $\pi \in K_{M}^{+}$, we define a map $\rho_{\pi}^{*}: L \rightarrow[-\infty, \infty]$ by setting

$$
\rho_{\pi}^{*}(x)=\sup _{\psi \in \operatorname{ext}(\pi)}\left(\sigma_{A}(\psi)-\psi(x)\right)=\sup _{\psi \in \operatorname{ext}(\pi) \cap \operatorname{bar}(A)}\left(\sigma_{A}(\psi)-\psi(x)\right) .
$$

Moreover, we define a map $u_{\pi}^{*}: L \rightarrow[-\infty, \infty]$ by setting

$$
u_{\pi}^{*}(x)=-\rho_{\pi}^{*}(x) \text {. }
$$

(If $A$ is a cone, then $\sigma_{A}=0$ on $\operatorname{bar}(A)$ and the above maps simplify accordingly).

The functionals $\rho_{\pi}^{*}$ are inspired by the dual representation of the risk measures $\rho_{\pi}$; see e.g. Frittelli and Scandolo [25] or Farkas et al. [20]. The precise link is shown in Proposition 4.14 below. For the time being, we are interested in highlighting some properties of the functionals $\rho_{\pi}^{*}$, or equivalently $u_{\pi}^{*}$, and proceeding to our desired multi-utility representation.

Proposition 4.8 For every $\pi \in K_{M}^{+}$, the functional $u_{\pi}^{*}$ has the following properties:

(i) $u_{\pi}^{*}$ is translative along $M$, i.e., for all $x \in L$ and $m \in M$,

$$
u_{\pi}^{*}(x+m)=u_{\pi}^{*}(x)+\pi(m) .
$$

(ii) $u_{\pi}^{*}$ is nondecreasing with respect to $\succeq_{K}$, i.e., for all $x, y \in L$,

$$
x \succeq_{K} y \quad \Longrightarrow \quad u_{\pi}^{*}(x) \geq u_{\pi}^{*}(y) .
$$

(iii) $u_{\pi}^{*}$ is concave, i.e., for all $x, y \in L$ and $\lambda \in[0,1]$,

$$
u_{\pi}^{*}(\lambda x+(1-\lambda) y) \geq \lambda u_{\pi}^{*}(x)+(1-\lambda) u_{\pi}^{*}(y) .
$$

(iv) $u_{\pi}^{*}$ is upper semicontinuous, i.e., for every net $\left(x_{\alpha}\right) \subseteq L$ and every $x \in L$,

$$
x_{\alpha} \rightarrow x \quad \Longrightarrow \quad \limsup u_{\pi}^{*}\left(x_{\alpha}\right) \geq u_{\pi}^{*}(x) .
$$

Proof Translativity follows from the definition of $\rho_{\pi}^{*}$. Being a supremum of affine maps, it is clear that $\rho_{\pi}^{*}$ is convex and lower semicontinuous. To show monotonicity, it suffices to observe that $\operatorname{bar}(A) \subseteq K^{+}$by Assumption 3.1, (A1) and therefore

$$
\rho_{\pi}^{*}(x)=\sup _{\psi \in \operatorname{ext}(\pi) \cap K^{+}}\left(\sigma_{A}(\psi)-\psi(x)\right) \leq \sup _{\psi \in \operatorname{ext}(\pi) \cap K^{+}}\left(\sigma_{A}(\psi)-\psi(y)\right)=\rho_{\pi}^{*}(y)
$$

for all $x, y \in L$ with $x \succeq_{K} y$, where we used that $\psi(x-y) \geq 0$ for every $\psi \in K^{+}$. 
To streamline the proof of the announced multi-utility representation, we start with the following lemma. We denote by $\operatorname{ker}(\pi)$ the kernel of $\pi \in M^{\prime}$, i.e.,

$$
\operatorname{ker}(\pi):=\{m \in M: \pi(m)=0\} .
$$

In the sequel, we repeatedly use the fact that $\operatorname{ker}(\pi)$ has codimension 1 in $M$ (provided $\pi$ is nonzero).

Lemma 4.9 The set A can be represented as

$$
A=\bigcap_{\pi \in K_{M}^{+} \backslash\{0\}} \operatorname{cl}(A+\operatorname{ker}(\pi)) .
$$

Moreover, for every $\pi \in K_{M}^{+}$, we have $\operatorname{bar}(\operatorname{cl}(A+\operatorname{ker}(\pi)))=\operatorname{bar}(A) \cap \operatorname{ker}(\pi)^{\perp}$ and

$$
\sigma_{\operatorname{cl}(A+\operatorname{ker}(\pi))}(\psi)= \begin{cases}\sigma_{A}(\psi) & \text { if } \psi \in \operatorname{ker}(\pi)^{\perp} \\ -\infty & \text { otherwise. }\end{cases}
$$

Proof We only prove the inclusion " $\supseteq$ " in (4.1) because the other assertions are clear. Assume that $x \in \operatorname{cl}(A+\operatorname{ker}(\pi))$ for every nonzero $\pi \in K_{M}^{+}$and take any $\psi \in \operatorname{bar}(A)$. In light of (2.1), to conclude the proof, we have to show that $\psi(x) \geq \sigma_{A}(\psi)$. To this effect, let $\pi_{\psi}$ be the restriction of $\psi$ to the space $M$. Since $\operatorname{bar}(A) \subseteq K^{+}$, it follows that $\pi_{\psi} \in K_{M}^{+}$. Moreover, note that $\psi \in \operatorname{ker}\left(\pi_{\psi}\right)^{\perp}$. As a result, we have

$$
\psi \in \operatorname{bar}(A) \cap \operatorname{ker}\left(\pi_{\psi}\right)^{\perp}=\operatorname{bar}\left(A+\operatorname{ker}\left(\pi_{\psi}\right)\right)=\operatorname{bar}\left(\operatorname{cl}\left(A+\operatorname{ker}\left(\pi_{\psi}\right)\right)\right) .
$$

Since $x \in \operatorname{cl}\left(A+\operatorname{ker}\left(\pi_{\psi}\right)\right)$ by our assumption, we can use (2.1) again to get

$$
\psi(x) \geq \sigma_{\mathrm{cl}\left(A+\operatorname{ker}\left(\pi_{\psi}\right)\right)}(\psi)=\sigma_{A+\operatorname{ker}\left(\pi_{\psi}\right)}(\psi)=\sigma_{A}(\psi),
$$

where the last equality holds because $\psi \in \operatorname{ker}\left(\pi_{\psi}\right)^{\perp}$. This concludes the proof.

The next lemma records a dual representation of the map $R$ that will immediately yield our desired multi-utility representation with (upper) semicontinuous functionals.

Lemma 4.10 For every $x \in L$, the set $R(x)$ can be represented as

$$
\begin{aligned}
R(x) & =\bigcap_{\pi \in K_{M}^{+} \backslash\{0\}}\left\{m \in M: \pi(m) \geq \rho_{\pi}^{*}(x)\right\} \\
& =\bigcap_{\pi \in K_{M}^{+} \backslash\{0\}} \bigcap_{\psi \in \operatorname{ext}(\pi)}\left\{m \in M: \pi(m) \geq \sigma_{A}(\psi)-\psi(x)\right\} .
\end{aligned}
$$

(If $A$ is a cone, then $\sigma_{A}=0$ on $\operatorname{bar}(A)$ and the representation simplifies accordingly). 
Proof Fix $x \in L$. It follows from the representation in (2.1) and Lemma 4.9 that

$$
R(x)=\bigcap_{\pi \in K_{M}^{+} \backslash\{0\}} \bigcap_{\psi \in \operatorname{ker}(\pi)^{\perp}}\left\{m \in M: \psi(m) \geq \sigma_{A}(\psi)-\psi(x)\right\} .
$$

To establish the desired representation of $R(x)$, it then suffices to show that the set $\operatorname{ker}(\pi)^{\perp}$ on the right-hand side of (4.2) can be replaced by $\operatorname{ext}(\pi)$. To this effect, let $m \in M$ satisfy $\pi(m) \geq \sigma_{A}(\psi)-\psi(x)$ for all nonzero $\pi \in K_{M}^{+}$and $\psi \in \operatorname{ext}(\pi)$. Moreover, take an arbitrary nonzero $\pi \in K_{M}^{+}$and an arbitrary $\psi \in \operatorname{ker}(\pi)^{\perp}$. To conclude the proof, we have to show that

$$
\psi(m) \geq \sigma_{A}(\psi)-\psi(x) .
$$

This is clear if $\psi \notin \operatorname{bar}(A)$ or $\psi \in \operatorname{ext}(\pi)$. Hence, assume that $\psi \in \operatorname{bar}(A) \backslash \operatorname{ext}(\pi)$. Note that since $\pi$ is nonzero and $K-K=L$, we find $n \in K_{M}$ such that $\pi(n)>0$. Since $\operatorname{bar}(A) \subseteq K^{+}$, two situations are possible. On the one hand, if $\psi(n)>0$, then $\psi$ belongs to $\operatorname{ext}(\pi)$ up to a strictly positive multiple and therefore (4.3) holds. On the other hand, if $\psi(n)=0$, then we must have $\psi \in M^{\perp}$. To deal with this case, note first that we always find a nonzero $\pi^{*} \in K_{M}^{+}$satisfying $\operatorname{ext}\left(\pi^{*}\right) \cap \operatorname{bar}(A) \neq \emptyset$, for otherwise every functional in $\operatorname{bar}(A) \cap \operatorname{ker}\left(\pi^{*}\right)^{\perp}$ would annihilate the entire $M$ and it would follow from (2.1) and (4.2) that $R(y)=M$ for every $y \in A$, which is against Proposition 3.2. Now, take $\varphi \in \operatorname{ext}\left(\pi^{*}\right) \cap \operatorname{bar}(A)$ and set $\varphi_{k}=\varphi+k \psi \in \operatorname{ext}\left(\pi^{*}\right)$ for each $k \in \mathbb{N}$. It follows that

$$
\begin{aligned}
\pi^{*}(m) & =\sup _{k \in \mathbb{N}} \varphi_{k}(m) \geq \sup _{k \in \mathbb{N}}\left(\sigma_{A}\left(\varphi_{k}\right)-\varphi_{k}(x)\right) \\
& \geq \sigma_{A}(\varphi)-\varphi(x)+\sup _{k \in \mathbb{N}}\left(k\left(\sigma_{A}(\psi)-\psi(x)\right)\right) .
\end{aligned}
$$

This implies that $\psi(m)=0 \geq \sigma_{A}(\psi)-\psi(x)$ must hold, establishing (4.3).

Theorem 4.11 The preference $\succeq_{R}$ can be represented by the multi-utility family

$$
\mathcal{U}^{*}=\left\{u_{\pi}^{*}: \pi \in K_{M}^{+} \backslash\{0\}, \operatorname{ext}(\pi) \cap \operatorname{bar}(A) \neq \emptyset\right\} .
$$

Proof Note that $\rho_{\pi}^{*}(x)=-\infty$ for every $x \in L$ whenever $\operatorname{ext}(\pi) \cap \operatorname{bar}(A)=\emptyset$ for some $\pi \in K_{M}^{+}$. Hence, the desired assertion follows immediately from Lemma 4.10; see also the proof of Theorem 4.5.

Remark 4.12 The statement of Lemma 4.10 provides a unifying formulation for the dual representations of set-valued risk measures in the literature. This is further illustrated in Sect. 5. The strategy used in the proof is different from the ones adopted in the literature which are often based on results from set-valued duality, thereby offering a complementary perspective on the existing proofs; see also Remark 4.15.

The next proposition shows the link between the two multi-utility representations we have established. In a sense made precise below, the representation $\mathcal{U}^{*}$ can be 
seen as the regularisation of $\mathcal{U}$ by means of (upper) semicontinuous hulls. Before we show this, it is useful to single out the following dual representation of the augmented acceptance set, which should be compared with Farkas et al. [20, Theorem 1].

Lemma 4.13 For every $\pi \in K_{M}^{+} \backslash\{0\}$ such that $\operatorname{ext}(\pi) \cap \operatorname{bar}(A) \neq \emptyset$, we have

$$
\operatorname{cl}(A+\operatorname{ker}(\pi))=\bigcap_{\psi \in \operatorname{ext}(\pi)}\left\{x \in L: \psi(x) \geq \sigma_{A}(\psi)\right\}
$$

Proof In view of (2.1) and Lemma 4.9, the assertion is equivalent to

$$
\bigcap_{\psi \in \operatorname{ker}(\pi)^{\perp}}\left\{x \in L: \psi(x) \geq \sigma_{A}(\psi)\right\}=\bigcap_{\psi \in \operatorname{ext}(\pi)}\left\{x \in L: \psi(x) \geq \sigma_{A}(\psi)\right\} .
$$

We only need to show the inclusion " $\supseteq$ ". To this end, we mimic the argument in the proof of Lemma 4.10. Let $x \in L$ belong to the right-hand side above and take $\psi \in \operatorname{ker}(\pi)^{\perp}$. We have to show that

$$
\psi(x) \geq \sigma_{A}(\psi)
$$

This is clear if $\psi \notin \operatorname{bar}(A)$ or $\psi \in \operatorname{ext}(\pi)$. Hence, assume that $\psi \in \operatorname{bar}(A) \backslash \operatorname{ext}(\pi)$. Note that since $\pi$ is nonzero and $K-K=L$, we find $n \in K_{M}$ such that $\pi(n)>0$. Since $\operatorname{bar}(A) \subseteq K^{+}$, two situations are possible. On the one hand, if $\psi(n)>0$, then $\psi$ belongs to $\operatorname{ext}(\pi)$ up to a strictly positive multiple and therefore (4.4) holds. On the other hand, if $\psi(n)=0$, then we must have $\psi \in M^{\perp}$. In this case, take any functional $\varphi \in \operatorname{ext}(\pi) \cap \operatorname{bar}(A)$ and set $\varphi_{k}=k \psi+\varphi \in \operatorname{ext}(\pi)$ for every $k \in \mathbb{N}$. Then

$$
\psi(x)+\frac{1}{k} \varphi(x)=\frac{1}{k} \varphi_{k}(x) \geq \frac{1}{k} \sigma_{A}\left(\varphi_{k}\right) \geq \sigma_{A}(\psi)+\frac{1}{k} \sigma_{A}(\varphi)
$$

for every $k \in \mathbb{N}$. Letting $k \rightarrow \infty$ yields (4.4) and concludes the proof.

For a given map $f: L \rightarrow[-\infty, \infty]$, we denote by $\operatorname{lsc}(f)$ the largest lower semicontinuous map dominated by $f$ and similarly by $\operatorname{usc}(f)$ the smallest upper semicontinuous map dominating $f$.

Proposition 4.14 For every $\pi \in K_{M}^{+} \backslash\{0\}$ such that $\operatorname{ext}(\pi) \cap \operatorname{bar}(A) \neq \emptyset$, the following statements hold:

(i) $\rho_{\pi}^{*}=\operatorname{lsc}\left(\rho_{\pi}\right)$.

(ii) $u_{\pi}^{*}=\operatorname{usc}\left(u_{\pi}\right)$.

Proof Fix a nonzero $\pi \in K_{M}^{+}$such that $\operatorname{ext}(\pi) \cap \operatorname{bar}(A) \neq \emptyset$. Clearly, we only need to show (i). To this effect, recall that $\rho_{\pi}^{*}$ is lower semicontinuous and note that it dominated by $\rho_{\pi}$. Indeed, for every $x \in L$ and for every $m \in M$ such that $x+m \in A$,

$$
\sup _{\psi \in \operatorname{ext}(\pi)}\left(\sigma_{A}(\psi)-\psi(x)\right) \leq \sup _{\psi \in \operatorname{ext}(\pi)}(\psi(x+m)-\psi(x))=\pi(m)
$$


showing $\rho_{\pi}^{*}(x) \leq \rho_{\pi}(x)$. Now take a lower semicontinuous $f: L \rightarrow[-\infty, \infty]$ such that $f \leq \rho_{\pi}$. We claim that $f \leq \rho_{\pi}^{*}$ as well. To show this, suppose to the contrary that $f(x)>\rho_{\pi}^{*}(x)$ for some $x \in L$. Note that

$$
\rho_{\pi}^{*}(x)=\inf \{\lambda \in \mathbb{R}: x+\lambda m \in \operatorname{cl}(A+\operatorname{ker}(\pi))\}
$$

by Lemma 4.13, where $m \in M$ is any element satisfying $\pi(m)=1$ (which exists because $\pi$ is nonzero). As a result, we must have $f(x)>\lambda$ for some $\lambda \in \mathbb{R}$ such that $x+\lambda m \in \operatorname{cl}(A+\operatorname{ker}(\pi))$. Hence, there exist two nets $\left(x_{\alpha}\right) \subseteq A$ and $\left(m_{\alpha}\right) \subseteq \operatorname{ker}(\pi)$ such that $x_{\alpha}+m_{\alpha} \rightarrow x+\lambda m$. Since $\{f>\lambda\}$ is open by lower semicontinuity, it eventually follows from the translativity of $\rho_{\pi}$ that

$$
\lambda<f\left(x_{\alpha}+m_{\alpha}-\lambda m\right) \leq \rho_{\pi}\left(x_{\alpha}+m_{\alpha}-\lambda m\right)=\rho_{\pi}\left(x_{\alpha}\right)+\lambda \leq \lambda .
$$

Since this is impossible, we infer that $f \leq \rho_{\pi}^{*}$ must hold, concluding the proof.

Remark 4.15 (i) The preceding proposition shows that the dual representation in Lemma 4.10 and hence the multi-utility representation in Theorem 4.11 can be equivalently stated in terms of the semicontinuous hulls of the functionals $\rho_{\pi}$ and $u_{\pi}$, respectively. This should be compared with the representation in Hamel and Heyde [28, Lemma 5.1].

(ii) The preceding proposition also suggests the following alternative path to establishing Lemma 4.10: (1) Start with the representation in Lemma 4.4. (2) Show that the functionals $\rho_{\pi}$ there can be replaced by their lower semicontinuous hulls $\operatorname{lsc}\left(\rho_{\pi}\right)$. (3) Show that we can discard from the representation all the functionals $\pi \in K_{M}^{+} \backslash\{0\}$ such that $\operatorname{lsc}\left(\rho_{\pi}\right)$ is not proper or, equivalently, $\operatorname{ext}(\pi) \cap \operatorname{bar}(A)=\emptyset$. (4) Use Proposition 4.14 to replace the functionals $\operatorname{lsc}\left(\rho_{\pi}\right)$ with the more explicit functionals $\rho_{\pi}^{*}$. The advantage of the strategy pursued in the proof of Lemma 4.10 is that it avoids passing through semicontinuous hulls and the analysis of their properness.

The representing functionals belonging to the multi-utility representation in Theorem 4.11 are by definition upper semicontinuous. As a final step, we want to find conditions ensuring a multi-utility representation consisting of continuous functionals only. To achieve this, we exploit the link between the functionals $\rho_{\pi}$ and their regularisations $\rho_{\pi}^{*}$ established in Proposition 4.14.

Lemma 4.16 Let $\pi \in K_{M}^{+} \backslash\{0\}$ be such that $\operatorname{ext}(\pi) \cap \operatorname{bar}(A) \neq \emptyset$. If $\operatorname{int}(A) \neq \emptyset$ and $\rho_{\pi}(x)<\infty$ for every $x \in L$, then $\rho_{\pi}$ is finite-valued and continuous. In particular, $\rho_{\pi}=\rho_{\pi}^{*}$.

Proof First of all, we claim that $\rho_{\pi}(x)>-\infty$ for every $x \in L$. To see this, take any functional $\psi \in \operatorname{ext}(\pi) \cap \operatorname{bar}(A)$ and note that for every $x \in L$,

$$
\rho_{\pi}(x) \geq \rho_{\pi}^{*}(x) \geq \sigma_{A}(\psi)-\psi(x)>-\infty .
$$

As a result, $\rho_{\pi}$ is finite-valued. Note that by definition, $\rho_{\pi}$ is bounded above on $A$ by 0 . Since $A$ has nonempty interior and $\rho_{\pi}$ is convex, we infer from Rockafel$\operatorname{lar}\left[40\right.$, Theorem 8] that $\rho_{\pi}$ is continuous. The last statement is a direct consequence of Proposition 4.14. 
The following multi-utility representation with continuous utility functionals is a direct consequence of Theorem 4.11 and Lemma 4.16.

Theorem 4.17 Assume that $\operatorname{int}(A) \neq \emptyset$ and that $\rho_{\pi}(x)<\infty$ for all $\pi \in K_{M}^{+} \backslash\{0\}$ with $\operatorname{ext}(\pi) \cap \operatorname{bar}(A) \neq \emptyset$ and $x \in L$. Then the preference $\succeq_{R}$ can be represented by the multi-utility family

$$
\mathcal{U}^{* *}=\left\{u_{\pi}: \pi \in K_{M}^{+} \backslash\{0\}, \operatorname{ext}(\pi) \cap \operatorname{bar}(A) \neq \emptyset\right\} .
$$

In addition, every element of $\mathcal{U}^{* *}$ is finite-valued and continuous.

We conclude by showing a number of sufficient conditions for the finiteness assumption in Lemma 4.16 to hold. This should be compared with the results in Farkas et al. [20, Sect. 3]. The recession cone of $A$ is denoted by

$$
\operatorname{rec}(A):=\{x \in L: x+y \in A, \forall y \in A\} .
$$

Note that $\operatorname{rec}(A)$ is the largest convex cone such that $A+\operatorname{rec}(A) \subseteq A$. In particular, if $A$ is a cone, then $\operatorname{rec}(A)=A$. Moreover, for any convex cone $C \subseteq L$, we denote by

$$
\operatorname{qint}(C):=\left\{x \in C: \psi(x)>0, \forall \psi \in C^{+} \backslash\{0\}\right\}
$$

the quasi-interior of $C$. Note that we always have $\operatorname{int}(C) \subseteq \operatorname{qint}(C)$.

Proposition 4.18 Let $\pi \in K_{M}^{+} \backslash\{0\}$ satisfy $\operatorname{ext}(\pi) \cap \operatorname{bar}(A) \neq \emptyset$. Then $\rho_{\pi}(x)<\infty$ for every $x \in L$ if any of the following conditions holds:

(i) $L=A+M$.

(ii) $M \cap \operatorname{qint}(K) \neq \emptyset$.

(iii) $M \cap \operatorname{qint}(\operatorname{rec}(A)) \neq \emptyset$.

Proof The desired assertion clearly holds under (i). Since $K \subseteq \operatorname{rec}(A)$ by Assumption 3.1, (A1), we see that $\operatorname{qint}(K) \subseteq \operatorname{qint}(\operatorname{rec}(A))$. Hence, it suffices to establish that (iii) implies the desired assertion. So assume that (iii) holds and take $m \in M \cap$ qint $(\operatorname{rec}(A))$. If $\rho_{\pi}(x)=\infty$ for some $x \in L$, then we must have $(x+M) \cap A=\emptyset$. It follows from a standard separation result, see e.g. Zălinescu [43, Theorem 1.1.3], that we find a nonzero functional $\psi \in L^{\prime}$ satisfying $\psi(x+\lambda m) \leq \sigma_{A}(\psi)$ for every $\lambda \in \mathbb{R}$. This is only possible if $\psi(m)=0$, which cannot hold because $\psi \in \operatorname{bar}(A) \subseteq(\operatorname{rec}(A))^{+}$. As a result, we must have $\rho_{\pi}(x)<\infty$ for every $x \in L$.

\section{Applications}

In this final section, we specify the general dual representation of $R$ to a number of concrete situations. The explicit formulation of the corresponding multi-utility representation can be easily derived as in Theorems 4.11 and 4.17. Throughout the section, we consider a probability space $(\Omega, \mathcal{F}, \mathbb{P})$ and fix an index $d \in \mathbb{N}$. For every 
$p \in[0, \infty]$ and every Borel-measurable set $S \subseteq \mathbb{R}^{d}$, we denote by $L^{p}(S)$ the set of all equivalence classes, with respect to almost-sure equality, of $d$-dimensional random vectors $X=\left(X_{1}, \ldots, X_{d}\right): \Omega \rightarrow \mathbb{R}^{d}$ with $p$-integrable components such that $\mathbb{P}[X \in S]=1$. As usual, we never explicitly distinguish between an equivalence class in $L^{p}(S)$ and any of its representative elements. We treat $\mathbb{R}^{d}$ as a linear subspace of $L^{p}\left(\mathbb{R}^{d}\right)$. For all vectors $a, b \in \mathbb{R}^{d}$, we set

$$
\langle a, b\rangle:=\sum_{i=1}^{d} a_{i} b_{i} .
$$

The expectation with respect to $\mathbb{P}$ is simply denoted by $\mathbb{E}$. For every $p \in[1, \infty]$, the space $L^{p}\left(\mathbb{R}^{d}\right)$ can be naturally paired with $L^{q}\left(\mathbb{R}^{d}\right)$ for $q=\frac{p}{p-1}$ via the bilinear form

$$
(X, Z) \mapsto \mathbb{E}[\langle X, Z\rangle]
$$

Here, we adopt the usual conventions $\frac{1}{0}:=\infty$ and $\frac{\infty}{\infty}:=1$. Finally, for every random vector $X \in L^{1}\left(\mathbb{R}^{d}\right)$, we use the compact notation

$$
\mathbb{E}[X]:=\left(\mathbb{E}\left[X_{1}\right], \ldots, \mathbb{E}\left[X_{d}\right]\right)
$$

\subsection{Set-valued risk measures in a multi-currency setting}

We consider a financial market where $d$ different currencies are traded. Every element of $L^{1}\left(\mathbb{R}^{d}\right)$ is interpreted as a vector of capital positions at some future point in time, expressed in our different currencies. For a pre-specified acceptance set $\mathcal{A} \subseteq L^{1}\left(\mathbb{R}^{d}\right)$, we look for the currency portfolios that have to be set up at the initial time to ensure acceptability.

\subsubsection{The static case}

As a first step, we consider a one-period market with dates 0 and 1 . In this setting, we focus on the currency portfolios that we have to build at time 0 in order to ensure acceptability of currency positions at time 1 . This naturally leads to defining the setvalued map $R: L^{1}\left(\mathbb{R}^{d}\right) \rightrightarrows \mathbb{R}^{d}$ by

$$
R(X):=\left\{m \in \mathbb{R}^{d}: X+m \in \mathcal{A}\right\} .
$$

Assumption 5.1 In this subsection, we work under the following assumptions:

(1) $\mathcal{A}$ is norm-closed, convex and satisfies $\mathcal{A}+L^{1}\left(\mathbb{R}_{+}^{d}\right) \subseteq \mathcal{A}$.

(2) $R(X) \notin\left\{\emptyset, \mathbb{R}^{d}\right\}$ for some $X \in L^{1}\left(\mathbb{R}^{d}\right)$.

We derive the following representation by applying our general results to

$$
\left(L, L^{\prime}, K, A, M\right)=\left(L^{1}\left(\mathbb{R}^{d}\right), L^{\infty}\left(\mathbb{R}^{d}\right), L^{1}\left(\mathbb{R}_{+}^{d}\right), \mathcal{A}, \mathbb{R}^{d}\right) .
$$

This result provides an $L^{1}$-version of the dual representation established in Jouini et al. [32], Kulikov [35] and Hamel and Heyde [28]. 
Proposition 5.2 For every $X \in L^{1}\left(\mathbb{R}^{d}\right)$, the set $R(X)$ can be represented as

$$
R(X)=\bigcap_{w \in \mathbb{R}_{+}^{d} \backslash\{0\}} \bigcap_{\substack{Z \in L^{\infty}\left(\mathbb{R}_{+}^{d}\right), \mathbb{E}[Z]=w}}\left\{m \in \mathbb{R}^{d}:\langle m, w\rangle \geq \sigma_{\mathcal{A}}(Z)-\mathbb{E}[\langle X, Z\rangle]\right\} .
$$

In addition, if $\mathcal{A}$ is a cone, we can simplify the above representation by using that

$$
\sigma_{\mathcal{A}}(Z)= \begin{cases}0 & \text { if } Z \in \mathcal{A}^{+}, \\ -\infty & \text { otherwise. }\end{cases}
$$

Proof Note that $K_{M}^{+}$can be identified with $\mathbb{R}_{+}^{d}$ and that $\operatorname{bar}(\mathcal{A})$ is contained in $L^{\infty}\left(\mathbb{R}_{+}^{d}\right)$ by Assumption 5.1, (1). Since for all $w \in \mathbb{R}^{d}$ and $Z \in L^{\infty}\left(\mathbb{R}^{d}\right)$, the random vector $Z$ (viewed as a functional on $L^{1}\left(\mathbb{R}^{d}\right)$ ) is an extension of $w$ (viewed as a functional on $\mathbb{R}^{d}$ ) precisely when $\mathbb{E}[Z]=w$, the desired representation follows immediately from Lemma 4.10.

Remark 5.3 Note that in the above framework, the set qint $(K)$ consists of all random vectors in $L^{1}\left(\mathbb{R}^{d}\right)$ whose components are strictly positive almost surely, and hence $M \cap$ qint $(K) \neq \emptyset$. This can be used to ensure multi-utility representations with continuous representing functionals; see Proposition 4.18.

Example 5.4 For every $X \in L^{1}(\mathbb{R})$ and every $\alpha \in(0,1)$, we denote by $\operatorname{ES}_{\alpha}(X)$ the expected shortfall of $X$ at level $\alpha$, i.e.,

$$
\operatorname{ES}_{\alpha}(X):=-\frac{1}{\alpha} \int_{0}^{\alpha} q_{X}(\beta) d \beta,
$$

where $q_{X}$ is any quantile function of $X$. The multi-dimensional acceptance set based on expected shortfall introduced in Hamel et al. [31] is given by

$$
\mathcal{A}=\left\{X \in L^{1}\left(\mathbb{R}^{d}\right): \operatorname{ES}_{\alpha_{i}}\left(X_{i}\right) \leq 0, \forall i \in\{1, \ldots, d\}\right\}
$$

for a fixed $\alpha=\left(\alpha_{1}, \ldots, \alpha_{d}\right) \in(0,1)^{d}$. Note that Assumptions 5.1, (1) and (2) hold. In particular, we have $R(0)=\mathbb{R}_{+}^{d}$. In addition, $\mathcal{A}$ is a cone. Note that

$$
\begin{aligned}
\mathcal{Z}_{w}^{\infty}(\alpha): & =\left\{Z \in L^{\infty}\left(\mathbb{R}^{d}\right): \mathbb{E}[Z]=w\right\} \cap \mathcal{A}^{+} \\
& =\left\{Z \in L^{\infty}\left(\mathbb{R}_{+}^{d}\right): \mathbb{E}[Z]=w, Z \leq \frac{w}{\alpha}\right\}
\end{aligned}
$$

for every $w \in \mathbb{R}_{+}^{d}$ (where $\frac{w}{\alpha}$ is understood component by component). This follows from the standard dual representation of expected shortfall; see Föllmer and Schied [24, Theorem 4.52]. As a result, the dual representation in Proposition 5.2 reads

$$
R(X)=\bigcap_{w \in \mathbb{R}_{+}^{d} \backslash\{0\}} \bigcap_{Z \in \mathcal{Z}_{w}^{\infty}(\alpha)}\left\{m \in \mathbb{R}^{d}:\langle m, w\rangle \geq-\mathbb{E}[\langle X, Z\rangle]\right\}
$$

for every random vector $X \in L^{1}\left(\mathbb{R}^{d}\right)$. 


\subsubsection{The dynamic case}

As a next step, we consider a multi-period financial market with dates $t=0, \ldots, T$ and information structure represented by a filtration $\left(\mathcal{F}_{t}\right)$ satisfying $\mathcal{F}_{0}=\{\emptyset, \Omega\}$ and $\mathcal{F}_{T}=\mathcal{F}$. In this setting, currency portfolios can be rebalanced through time. A (random) portfolio at time $t \in\{0, \ldots, T\}$ is represented by an $\mathcal{F}_{t}$-measurable random vector in $L^{0}\left(\mathbb{R}^{d}\right)$. We denote by $\mathcal{C}_{t}$ the set of $\mathcal{F}_{t}$-measurable portfolios that can be converted into portfolios with nonnegative components by trading at time $t$. This means that for all $\mathcal{F}_{t}$-measurable portfolios $m_{t}$ and $n_{t}$, we can exchange $m_{t}$ for $n_{t}$ at time $t$ provided that $m_{t}-n_{t} \in \mathcal{C}_{t}$. The sets $\mathcal{C}_{t}$ are meant to capture potential transaction costs. A flow of portfolios is represented by an adapted process $\left(m_{t}\right)$. More precisely, for every date $t \in\{0, \ldots, T-1\}$, the portfolio $m_{t}$ is set up at time $t$ and held until time $t+1$. The portfolio flows belonging to the set

$$
\mathcal{C}:=\left\{\left(m_{t}\right): m_{t}-m_{t+1} \in \mathcal{C}_{t+1}, \forall t \in\{0, \ldots, T-1\}\right\}
$$

are said to be admissible. The admissibility condition is a direct extension of the standard self-financing property in frictionless markets.

We look for all initial portfolios that can be rebalanced in an admissible way until the terminal date in order to ensure acceptability. This leads to the set-valued map $R: L^{1}\left(\mathbb{R}^{d}\right) \rightrightarrows \mathbb{R}^{d}$ defined by

$$
\begin{aligned}
R(X):=\left\{m \in \mathbb{R}^{d}:\right. & \exists\left(m_{t}\right) \in \mathcal{C}, n_{T} \in L^{0}\left(\mathbb{R}^{d}\right) \text { with } \\
& \left.m-m_{0} \in \mathcal{C}_{0}, m_{T}-n_{T} \in \mathcal{C}_{T}, X+n_{T} \in \mathcal{A}\right\} .
\end{aligned}
$$

In words, the above set consists of all initial portfolios that give rise, after a convenient exchange at date 0 , to an admissible rebalancing process making the outstanding currency position acceptable after a final portfolio adjustment at time $T$. This setting can be embedded in our framework because we can equivalently write

$$
R(X)=\left\{m \in \mathbb{R}^{d}: X+m \in \mathcal{A}+\sum_{t=0}^{T} \mathcal{C}_{t}\right\}
$$

Assumption 5.5 In this subsection, we work under the following assumptions:

(1) $\mathcal{A}$ is norm-closed, convex and satisfies $\mathcal{A}+L^{1}\left(\mathbb{R}_{+}^{d}\right) \subseteq \mathcal{A}$.

(2) $\mathcal{C}_{t}$ is convex and contains $L_{t}^{0}\left(\mathbb{R}_{+}^{d}\right)$ for every $t \in\{0, \ldots, T\}$.

(3) $\left(\mathcal{A}+\sum_{t=0}^{T} \mathcal{C}_{t}\right) \cap L^{1}\left(\mathbb{R}^{d}\right)$ is norm-closed.

(4) $R(X) \notin\left\{\emptyset, \mathbb{R}^{d}\right\}$ for some $X \in L^{1}\left(\mathbb{R}^{d}\right)$.

We derive the following representation by applying our general results to

$\left(L, L^{\prime}, K, A, M\right)=\left(L^{1}\left(\mathbb{R}^{d}\right), L^{\infty}\left(\mathbb{R}^{d}\right), L^{1}\left(\mathbb{R}_{+}^{d}\right),\left(\mathcal{A}+\sum_{t=0}^{T} \mathcal{C}_{t}\right) \cap L^{1}\left(\mathbb{R}^{d}\right), \mathbb{R}^{d}\right)$ 
For convenience, we also set

$$
\mathcal{C}_{1: T}^{1}:=\left(\sum_{t=1}^{T} \mathcal{C}_{t}\right) \cap L^{1}\left(\mathbb{R}^{d}\right)
$$

For later use, note that

$$
\operatorname{bar}\left(\mathcal{C}_{1: T}^{1}\right) \subseteq \operatorname{bar}\left(\sum_{t=1}^{T}\left(\mathcal{C}_{t} \cap L^{1}\left(\mathbb{R}^{d}\right)\right)\right)=\bigcap_{t=1}^{T} \operatorname{bar}\left(\mathcal{C}_{t} \cap L^{1}\left(\mathbb{R}^{d}\right)\right)
$$

The next result provides an $L^{1}$-version of the dual representation established in Hamel et al. [30] in the special setting of Example 5.8.

Proposition 5.6 For every $X \in L^{1}\left(\mathbb{R}^{d}\right)$, the set $R(X)$ can be represented as

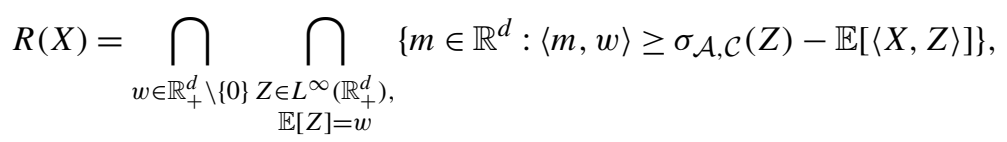

where we have set for every $Z \in L^{\infty}\left(\mathbb{R}^{d}\right)$

$$
\sigma_{\mathcal{A}, \mathcal{C}}(Z):=\sigma_{\mathcal{A}}(Z)+\sigma_{\mathcal{C}_{0}}(Z)+\sigma_{\mathcal{C}_{1: T}^{1}}(Z)
$$

In addition, if $\mathcal{A}$ is a cone, the above representation can be simplified by using that

$$
\sigma_{\mathcal{A}}(Z)= \begin{cases}0 & \text { if } Z \in \mathcal{A}^{+}, \\ -\infty & \text { otherwise }\end{cases}
$$

Moreover, if $\mathcal{C}_{0}$ is a cone, then

$$
\sigma_{\mathcal{C}_{0}}(Z)= \begin{cases}0 & \text { if } Z \in L^{\infty}\left(\mathbb{R}_{+}^{d}\right), \mathbb{E}[Z] \in \mathcal{C}_{0}^{+} \\ -\infty & \text { otherwise }\end{cases}
$$

Similarly, if $\mathcal{C}_{t}$ is a cone for every $t \in\{1, \ldots, T\}$, then

$$
\sigma_{\mathcal{C}_{1: T}^{1}}(Z)= \begin{cases}0 & \text { if } Z \in\left(\mathcal{C}_{1: T}^{1}\right)^{+} \subseteq \bigcap_{t=1}^{T}\left(\mathcal{C}_{t} \cap L^{1}\left(\mathbb{R}^{d}\right)\right)^{+}, \\ -\infty & \text { otherwise }\end{cases}
$$

Proof The assertion follows from Proposition 5.2 because $\mathcal{C}_{0} \subseteq \mathbb{R}^{d}$ implies

$$
\left(\mathcal{A}+\sum_{t=0}^{T} \mathcal{C}_{t}\right) \cap L^{1}\left(\mathbb{R}^{d}\right)=\mathcal{A}+\mathcal{C}_{0}+\mathcal{C}_{1: T}^{1}
$$

and we observe that $\sigma_{\mathcal{A}+\mathcal{C}_{0}+\mathcal{C}_{1: T}^{1}}=\sigma_{\mathcal{A}}+\sigma_{\mathcal{C}_{0}}+\sigma_{\mathcal{C}_{1: T}^{1}}$. 
Remark 5.7 Note that as in the static case, we have $M \cap \operatorname{qint}(K) \neq \emptyset$. This can be used to ensure multi-utility representations with continuous representing functionals; see Proposition 4.18.

Example 5.8 We consider superreplication under proportional transaction costs and adopt the discrete version of the model by Kabanov [33]. For every $t \in\{0, \ldots, T\}$, we say that a set-valued map $S: \Omega \rightrightarrows \mathbb{R}^{d}$ is $\mathcal{F}_{t}$-measurable provided that

$$
\{\omega \in \Omega: S(\omega) \cap \mathcal{U} \neq \emptyset\} \in \mathcal{F}_{t}
$$

for every open set $\mathcal{U} \subseteq \mathbb{R}^{d}$. In this case, we denote by $L^{0}(S)$ the set of all random vectors $X \in L^{0}\left(\mathbb{R}^{d}\right)$ such that $\mathbb{P}[X \in S]=1$. This set is always nonempty if $S$ has closed values; see Rockafellar and Wets [41, Corollary 14.6]. Now let $K_{t}: \Omega \rightrightarrows \mathbb{R}^{d}$ be an $\mathcal{F}_{t}$-measurable set-valued map such that $K_{t}(\omega)$ is a polyhedral convex cone (hence $K_{t}(\omega)$ is closed) containing $\mathbb{R}_{+}^{d}$ for every $\omega \in \Omega$ and set

$$
\mathcal{C}_{t}=L^{0}\left(K_{t}\right)
$$

Moreover, we consider the worst-case acceptance set

$$
\mathcal{A}=L^{1}\left(\mathbb{R}_{+}^{d}\right) .
$$

Assumptions 5.5, (1) and (2) are easily seen to be satisfied. Moreover, $\mathcal{A}$ as well as each of the sets $\mathcal{C}_{t}$ is a cone. As proved in Schachermayer [42, Theorem 2.1], Assumption 5.5, (3) always holds under the so-called "robust no-arbitrage" condition. Finally, as $0 \in R(0)$, Assumption 5.5, (4) holds if and only if $\mathbb{R}^{d}$ is not entirely contained in $\sum_{t=0}^{T} \mathcal{C}_{t}$. Note also that $\mathcal{A}^{+}=L^{\infty}\left(\mathbb{R}_{+}^{d}\right)$. As a result, Proposition 5.6 yields

$$
R(X)=\bigcap_{w \in K_{0}^{+} \backslash\{0\}} \bigcap_{\substack{Z \in L^{\infty}\left(\mathbb{R}_{+}^{d}\right), Z \in\left(\mathcal{C}_{1: T}^{1}\right)^{+}, \mathbb{E}[Z]=w}}\left\{m \in \mathbb{R}^{d}:\langle m, w\rangle \geq-\mathbb{E}[\langle X, Z\rangle]\right\}
$$

for every $X \in L^{1}\left(\mathbb{R}^{d}\right)$. The dual elements $Z$ in the above representation can be linked to consistent pricing systems; see e.g. Schachermayer [42]. To see this, note that for every $t \in\{0, \ldots, T\}$, the set-valued map $K_{t}^{+}: \Omega \rightrightarrows \mathbb{R}^{d}$ defined by

$$
K_{t}^{+}(\omega)=\left(K_{t}(\omega)\right)^{+}
$$

is $\mathcal{F}_{t}$-measurable, see e.g. Rockafellar and Wets [41, Exercise 14.12], and such that

$$
\left(\mathcal{C}_{t} \cap L^{1}\left(\mathbb{R}^{d}\right)\right)^{+}=L^{0}\left(K_{t}^{+}\right) \cap L^{\infty}\left(\mathbb{R}^{d}\right)
$$

by measurable selection; see the argument in the proof of Schachermayer [42, Theorem 1.7]. As a result, every dual element $Z$ in the above dual representation satisfies

$$
\mathbb{E}[Z] \in K_{0}^{+}, \quad Z \in\left(\mathcal{C}_{1: T}^{1}\right)^{+} \subseteq \bigcap_{t=1}^{T}\left(\mathcal{C}_{t} \cap L^{1}\left(\mathbb{R}^{d}\right)\right)^{+} \subseteq \bigcap_{t=1}^{T} L^{0}\left(K_{t}^{+}\right) .
$$


This shows that the $d$-dimensional adapted process $\left(\mathbb{E}\left[Z \mid \mathcal{F}_{t}\right]\right)$, with the conditional expectations taken componentwise, satisfies $\mathbb{E}\left[Z \mid \mathcal{F}_{T}\right]=Z$ and $\mathbb{E}\left[Z \mid \mathcal{F}_{t}\right] \in L^{0}\left(K_{t}^{+}\right)$ for every $t \in\{0, \ldots, T\}$ and thus qualifies as a consistent pricing system. In other words, the above dual elements $Z$ can be viewed as the terminal values of consistent pricing systems.

Remark 5.9 (i) It is worth noting that our approach provides a different path, compared to the strategy pursued in Schachermayer [42], to establish the existence of consistent pricing systems under the robust no-arbitrage assumption (admitting the closedness of the reference target set). Moreover, by rewriting the above dual representation in terms of consistent pricing systems, we recover the (localisation to $L^{1}\left(\mathbb{R}^{d}\right)$ of the) superreplication theorem by Schachermayer [42].

(ii) The above dual representation was also obtained in Hamel et al. [30]. Differently from that paper, we have not derived it from the superreplication theorem in Schachermayer [42], but from a direct application of our general results.

\subsection{Systemic set-valued risk measures based on acceptance sets}

We consider a single-period economy with dates 0 and 1 and a financial system consisting of $d$ entities. Every element of $L^{\infty}\left(\mathbb{R}^{d}\right)$ is interpreted as a vector of capital positions at time 1 of the various financial entities. The individual positions can be aggregated through a function $\Lambda: \mathbb{R}^{d} \rightarrow \mathbb{R}$. For a pre-specified acceptance set $\mathcal{A} \subseteq L^{\infty}(\mathbb{R})$, we look for the cash injections at time 0 that ensure the acceptability of the aggregated system. This leads to the set-valued map $R: L^{\infty}\left(\mathbb{R}^{d}\right) \rightrightarrows \mathbb{R}^{d}$ defined by

$$
R(X):=\left\{m \in \mathbb{R}^{d}: \Lambda(X+m) \in \mathcal{A}\right\} .
$$

This setting can be easily embedded in our framework because we can write

$$
R(X)=\left\{m \in \mathbb{R}^{d}: X+m \in \Lambda^{-1}(\mathcal{A})\right\}
$$

Assumption 5.10 In this subsection, we work under the following assumptions:

(1) $\mathcal{A}$ is convex and satisfies $\mathcal{A}+L^{\infty}\left(\mathbb{R}_{+}\right) \subseteq \mathcal{A}$.

(2) $\Lambda$ is nondecreasing and concave.

(3) $\Lambda^{-1}(\mathcal{A})$ is $\sigma\left(L^{\infty}\left(\mathbb{R}^{d}\right), L^{1}\left(\mathbb{R}^{d}\right)\right)$-closed.

(4) $R(X) \notin\left\{\emptyset, \mathbb{R}^{d}\right\}$ for some $X \in L^{\infty}\left(\mathbb{R}^{d}\right)$.

We derive the following representation by applying our general results to

$$
\left(L, L^{\prime}, K, A, M\right)=\left(L^{\infty}\left(\mathbb{R}^{d}\right), L^{1}\left(\mathbb{R}^{d}\right), L^{\infty}\left(\mathbb{R}_{+}^{d}\right), \Lambda^{-1}(\mathcal{A}), \mathbb{R}^{d}\right) .
$$

The result provides an equivalent formulation of the dual representation of sensitive systemic set-valued risk measures established in Ararat and Rudloff [1]. 
Proposition 5.11 For every $X \in L^{\infty}\left(\mathbb{R}^{d}\right)$, the set $R(X)$ can be represented as

$$
R(X)=\bigcap_{w \in \mathbb{R}_{+}^{d} \backslash\{0\}} \bigcap_{\begin{array}{c}
Z \in L^{1}\left(\mathbb{R}_{+}^{d}\right), \\
\mathbb{E}[Z]=w
\end{array}}\left\{m \in \mathbb{R}^{d}:\langle m, w\rangle \geq \sigma_{\Lambda^{-1}(\mathcal{A})}(Z)-\mathbb{E}[\langle X, Z\rangle]\right\}
$$

If $\Lambda^{-1}(\mathcal{A})$ is a cone, we can simplify the above representation by using that

$$
\sigma_{\Lambda^{-1}(\mathcal{A})}(Z)= \begin{cases}0 & \text { if } Z \in\left(\Lambda^{-1}(\mathcal{A})\right)^{+} \\ -\infty & \text { otherwise }\end{cases}
$$

Proof Note that $\Lambda^{-1}(\mathcal{A})$ is convex and satisfies $\Lambda^{-1}(\mathcal{A})+L^{\infty}\left(\mathbb{R}_{+}^{d}\right) \subseteq \Lambda^{-1}(\mathcal{A})$ by Assumption 5.10, (1) and (2). Note also that $K_{M}^{+}$can be identified with $\mathbb{R}_{+}^{d}$. In addition, we have $\operatorname{bar}\left(\Lambda^{-1}(\mathcal{A})\right) \subseteq L^{1}\left(\mathbb{R}_{+}^{d}\right)$. Since for all $w \in \mathbb{R}^{d}$ and $Z \in L^{1}\left(\mathbb{R}^{d}\right)$, the random vector $Z$ (viewed as a functional on $L^{\infty}\left(\mathbb{R}^{d}\right)$ ) is an extension of $w$ (viewed as a functional on $\mathbb{R}^{d}$ ) precisely when $\mathbb{E}[Z]=w$, the desired representation follows from Lemma 4.10.

Example 5.12 Let $\alpha=\left(\alpha_{1}, \ldots, \alpha_{d}\right) \in(1, \infty)^{d}$ and consider the aggregation function $\Lambda: \mathbb{R}^{d} \rightarrow \mathbb{R}$ defined by

$$
\Lambda(x)=\sum_{i=1}^{d} \max \left(x_{i}, 0\right)+\sum_{i=1}^{d} \alpha_{i} \min \left(x_{i}, 0\right) .
$$

Moreover, define the acceptance set $\mathcal{A} \subseteq L^{\infty}(\mathbb{R})$ by

$$
\mathcal{A}=\left\{X \in L^{\infty}(\mathbb{R}): \mathbb{E}[X] \geq 0\right\} .
$$

Clearly, Assumption 5.10, (1) and (2) hold. Since $\mathcal{A}$ is closed with respect to dominated almost sure convergence and $\Lambda$ is continuous, we easily see that $\Lambda^{-1}(\mathcal{A})$ is also closed with respect to dominated almost sure convergence. Hence we can repeat the argument in Delbaen [12, Theorem 3.2] to conclude that Assumption 5.10, (3) holds; see also Arduca et al. [2, Proposition 3.2]. Finally, note that $R(0)=\left\{m \in \mathbb{R}^{d}: \Lambda(m) \geq 0\right\}$, showing that Assumption 5.10, (4) holds. Now observe that $\operatorname{bar}(\mathcal{A})=\mathbb{R}_{+}$. The concave conjugate of $\Lambda$ is the map $\Lambda^{\bullet}: \mathbb{R}^{d} \rightarrow[-\infty, \infty)$ defined by

$$
\Lambda^{\bullet}(z):=\inf _{x \in \mathbb{R}^{d}}(\langle x, z\rangle-\Lambda(x))
$$

It is easy to verify that

$$
\Lambda^{\bullet}(z)= \begin{cases}0 & \text { if } z \in\left[1, \alpha_{1}\right] \times \cdots \times\left[1, \alpha_{d}\right], \\ -\infty & \text { otherwise. }\end{cases}
$$

Then it follows from Arduca et al. [2, Proposition 3.13 and Lemma 3.20] that

$$
\sigma_{\Lambda^{-1}(\mathcal{A})}(Z)= \begin{cases}0 & \text { if there exists } \lambda \in[0, \infty) \text { such that } \lambda e \leq Z \leq \lambda \alpha, \\ -\infty & \text { otherwise, }\end{cases}
$$


where $e=(1, \ldots, 1) \in \mathbb{R}^{d}$. Now for notational convenience, define

$$
\mathcal{Z}^{\infty}(\alpha):=\left\{Z \in L^{\infty}\left(\mathbb{R}_{+}^{d}\right): \exists \lambda \in[0, \infty) \text { with } \lambda e \leq Z \leq \lambda \alpha\right\}
$$

As a result, Proposition 5.11 yields

$$
R(X)=\bigcap_{w \in \mathbb{R}_{+}^{d} \backslash\{0\}} \bigcap_{\substack{Z \in \mathcal{Z}^{\infty}(\alpha), \mathbb{E}[Z]=w}}\left\{m \in \mathbb{R}^{d}:\langle m, w\rangle \geq-\mathbb{E}[\langle X, Z\rangle]\right\}
$$

for every random vector $X \in L^{\infty}\left(\mathbb{R}^{d}\right)$.

\section{Conclusions}

In this note, we establish a variety of representations for set-valued risk measures and show how to use them in order to derive suitable numerical representations for the induced incomplete preferences. Special attention is devoted to obtaining numerical representations expressed in terms of (semi)continuous utility functionals. The most appealing feature of such representing functionals is that they coincide, up to a sign, with scalar risk measures based on acceptance sets, which have been the subject of intense research in the past years. This demonstrates that under mild assumptions, a set-valued risk measure can be completely characterised by way of familiar scalar risk measures. In the last section, we illustrate our general results in the context of multi-currency markets and systemic risk. A natural direction of future research is the study of (set-valued) optimisation problems, e.g. portfolio selection or capital allocation, where the incomplete preference induced by a set-valued risk measure appears as a constraint. This is in the spirit of the applications discussed in the recent work by Crespi et al. [11]. Another interesting direction is to clarify the connection between the incomplete preferences studied in this note and the notion of time-consistency for dynamic set-valued risk measures studied e.g. in Feinstein and Rudloff [21], which can be viewed as a form of persistence of the preference relation through time. This link was kindly pointed out by a referee during the review process.

Funding Note Open access funding provided by University of Zurich.

Publisher's Note Springer Nature remains neutral with regard to jurisdictional claims in published maps and institutional affiliations.

Open Access This article is licensed under a Creative Commons Attribution 4.0 International License, which permits use, sharing, adaptation, distribution and reproduction in any medium or format, as long as you give appropriate credit to the original author(s) and the source, provide a link to the Creative Commons licence, and indicate if changes were made. The images or other third party material in this article are included in the article's Creative Commons licence, unless indicated otherwise in a credit line to the material. If material is not included in the article's Creative Commons licence and your intended use is not permitted by statutory regulation or exceeds the permitted use, you will need to obtain permission directly from the copyright holder. To view a copy of this licence, visit http://creativecommons.org/licenses/by/4.0/. 


\section{References}

1. Ararat, Ç., Rudloff, B.: Dual representations for systemic risk measures. Math. Financ. Econ. 14, 139-174 (2020)

2. Arduca, M., Koch-Medina, P., Munari, C.: Dual representations for systemic risk measures based on acceptance sets. Math. Financ. Econ. (2021). https://doi.org/10.1007/s11579-019-00250-0

3. Armenti, Y., Crépey, S., Drapeau, S., Papapantoleon, A.: Multivariate shortfall risk allocation and systemic risk. SIAM J. Financ. Math. 9, 90-126 (2018)

4. Aubin, J.-P., Ekeland, I.: Applied Nonlinear Analysis. Dover, New York (2006)

5. Aumann, R.J.: Utility theory without the completeness axiom. Econometrica 30, 445-462 (1962)

6. Baes, M., Koch-Medina, P., Munari, C.: Existence, uniqueness, and stability of optimal payoffs of eligible assets. Math. Finance 30, 128-166 (2020)

7. Bevilacqua, P., Bosi, G., Kaucic, M., Zuanon, M.E.: Pareto optimality on compact spaces in a preference-based setting under incompleteness. Int. J. Uncertain. Fuzziness 27, 239-249 (2019)

8. Bewley, T.F.: Knightian decision theory. Part I. Decis. Econ. Finance 25, 79-110 (2002)

9. Bosi, G., Estevan, A., Zuanon, M.E.: Partial representations of orderings. Int. J. Uncertain. Fuzziness 26, 453-473 (2018)

10. Bosi, G., Herden, G.: Continuous multi-utility representations of preorders. J. Math. Econ. 48, 212218 (2012)

11. Crespi, G., Hamel, A.H., Rocca, M., Schrage, C.: Set relations and approximate solutions in set optimization. Working paper (2018). Available online at https://arxiv.org/abs/1812.03300

12. Delbaen, F.: Coherent risk measures on general probability spaces. In: Sandmann, K., Schönbucher, P.J. (eds.) Advances in Finance and Stochastics: Essays in Honour of Dieter Sondermann, pp. 1-37. Springer, Berlin (2002)

13. Drapeau, S., Kupper, M.: Risk preferences and their robust representations. Math. Oper. Res. 38, 28-62 (2013)

14. Dubra, J., Maccheroni, F., Ok, E.A.: Expected utility theory without the completeness axiom. J. Econ. Theory 115, 118-133 (2004)

15. Eliaz, K., Ok, E.A.: Indifference or indecisiveness? Choice-theoretic foundations of incomplete preferences. Games Econ. Behav. 56, 61-86 (2006)

16. Evren, Ö.: On the existence of expected multi-utility representations. Econ. Theory 35, 575-592 (2008)

17. Evren, Ö.: Scalarization methods and expected multi-utility representations. J. Econ. Theory 151, 30-63 (2014)

18. Evren, Ö., Ok, E.A.: On the multi-utility representation of preference relations. J. Math. Econ. 47, 554-563 (2011)

19. Farkas, W., Koch-Medina, P., Munari, C.: Beyond cash-additive risk measures: when changing the numéraire fails. Finance Stoch. 18, 145-173 (2014)

20. Farkas, W., Koch-Medina, P., Munari, C.: Measuring risk with multiple eligible assets. Math. Financ. Econ. 9, 3-27 (2015)

21. Feinstein, Z., Rudloff, B.: Time consistency of dynamic risk measures in markets with transaction costs. Quant. Finance 13, 1473-1489 (2013)

22. Feinstein, Z., Rudloff, B., Weber, S.: Measures of systemic risk. SIAM J. Financ. Math. 8, 672-708 (2017)

23. Föllmer, H., Schied, A.: Convex measures of risk and trading constraints. Finance Stoch. 6, 429-447 (2002)

24. Föllmer, H., Schied, A.: Stochastic Finance: An Introduction in Discrete Time, 4th edn. de Gruyter, Berlin (2016)

25. Frittelli, M., Scandolo, G.: Risk measures and capital requirements for processes. Math. Finance 16, 589-612 (2006)

26. Galaabaatar, T., Karni, E.: Subjective expected utility with incomplete preferences. Econometrica 81, 255-284 (2013)

27. Haier, A., Molchanov, I., Schmutz, M.: Intragroup transfers, intragroup diversification and their risk assessment. Ann. Finance 12, 363-392 (2016)

28. Hamel, A.H., Heyde, F.: Duality for set-valued measures of risk. SIAM J. Financ. Math. 1, 66-95 (2010)

29. Hamel, A.H., Heyde, F., Löhne, A., Rudloff, B., Schrage, C.: Set optimization. A rather short introduction. In: Hamel, A., et al. (eds.) Set Optimization and Applications: The State of the Art, pp. 65-141. Springer, Berlin (2015) 
30. Hamel, A.H., Heyde, F., Rudloff, B.: Set-valued risk measures for conical market models. Math. Financ. Econ. 5, 1-28 (2011)

31. Hamel, A.H., Rudloff, B., Yankova, M.: Set-valued average value at risk and its computation. Math. Financ. Econ. 7, 229-246 (2013)

32. Jouini, E., Meddeb, M., Touzi, N.: Vector-valued coherent risk measures. Finance Stoch. 8, 531-552 (2004)

33. Kabanov, Y.: Hedging and liquidation under transaction costs in currency markets. Finance Stoch. 3, 237-248 (1999)

34. Kaminski, B.: On quasi-orderings and multi-objective functions. Eur. J. Oper. Res. 177, 1591-1598 (2007)

35. Kulikov, A.V.: Multidimensional coherent and convex risk measures. Theory Probab. Appl. 52, 614$635(2008)$

36. Mandler, M.: Incomplete preferences and rational intransitivity of choice. Games Econ. Behav. 50, 255-277 (2005)

37. Molchanov, I., Cascos, I.: Multivariate risk measures: a constructive approach based on selections. Math. Finance 26, 867-900 (2016)

38. Nishimura, H., Ok, E.A.: Utility representation of an incomplete and nontransitive preference relation. J. Econ. Theory 166, 164-185 (2016)

39. Ok, E.A.: Utility representation of an incomplete preference relation. J. Econ. Theory 104, 429-449 (2002)

40. Rockafellar, R.T.: Conjugate Duality and Optimization. SIAM, Philadelphia (1974)

41. Rockafellar, R.T., Wets, R.J.-B.: Variational Analysis. Springer, Berlin (2009)

42. Schachermayer, W.: The fundamental theorem of asset pricing under proportional transaction costs in finite discrete time. Math. Finance 14, 19-48 (2004)

43. Zălinescu, C.: Convex Analysis in General Vector Spaces. World Scientific, Singapore (2002) 